\title{
Core polarization, Brown-Rho scaling and a memory of Gerry's Princeton Years
}

\author{
T. T. S. Kuo \\ Department of Physics, State University New York, Stony Brook, New York 11794, USA \\ J. W. Holt \\ Department of Physics, University of Washington, Seattle, WA 98195, USA
}

(Dated: October 4, 2018)

\begin{abstract}
Core-polarization (CP) and Brown-Rho (BR) scaling were among Gerry's most favorite topics. In this contribution, we will discuss some of the early history as well as more recent work associated with these two fascinating phenomena. I (TTSK) will begin with some recollections of Princeton, where I met Gerry for the first time in 1964 and worked as his postdoc. Core polarization was in fact the first topic he assigned to me. JWH started working with Gerry at Stony Brook from 2003 and was Gerry's last student in nuclear physics. We three had teamed up well, working closely on both CP and BR scaling, particularly on the latter's connection to the anomalously-long beta-decay lifetime of carbon-14. We shall here briefly review these topics, including a recently developed new Brown-Rho scaling based on a Skyrmion half-Skyrmion two-phase model.

PACS numbers:
\end{abstract}

\section{INTRODUCTION}

This is a tribute to Gerry Brown who was our mentor, collaborator and good friend for a long long time, from 1964 to 2013 for TTSK, and from 2003 to 2013 for JWH. We remember him and our times together very well, and here we shall review briefly two subjects, 'core polarization' and 'Brown-Rho scaling', on which we have collaborated extensively over the years. Before doing so, we think we should first describe a recent book project [1] we worked on together:

Gerry talked with me (TTSK) one day in early 2007 about writing a book on 'Nucleon-Nucleon Interactions and the Nuclear Many-Body Problem'. Gerry's idea was to put together a reprint volume with a few introductory chapters and a collection of our published works spanning a period of about forty years. I of course was very happy about the idea, and soon afterward WorldScientific (WS) agreed to support the project. Gerry then asked JWH (then finishing up his Ph.D with Gerry) and Prof. Sabine Lee (Univ. of Birmingham) to help with the writing, typsetting (LaTexing) and organization of the book. (Gerry often said "The young are supposed to help the old.") As usual, Gerry was a man of quick action. In the summer of 2007 we had meetings at Gerry's home, and a tentative plan of 'who-does-what' was laid. So we started to work. Gerry in fact quickly wrote many neatly hand-written pages and sent us copies of them. Gerry often said he had only a 5-dollar pocket calculator, a sort of excuse he used to avoid doing calculations on computers and learning to use LaTex. So Sabine nicely typset all of Gerry's hand-written notes, and attached below is what Gerry wrote, in his unique style, in a memorable preface for the book:

Preface: Why now is a good time to write about the Nucleon-Nucleon Interaction and the Nuclear

\section{Many Body Problem}

"Why do two old nuclear physicists, with the help of a junior colleague and a historian, now write about the nucleon-nucleon interaction to which they have devoted such a large portion of their research lives previously?

The immediate explanation is straightforward. The main problems at the level of meson exchange physics have been solved. We now have an effective nucleonnucleon interaction $V_{l o w-k}$, pioneered in a renormalization group formalism by several of us at Stony Brook and our colleagues at Naples, which is nearly universally accepted as the unique low-momentum interaction that includes all experimental information to date.

Why does this make reconstructing the history of our understanding of the nucleon-nucleon interaction necessary or useful? There are several good reasons for engaging in a historical appreciation of the progression of research and the developments leading to our current knowledge in this subject area.

First, our understanding is based on a multi-step development in which a variety of different scientific insights and a wide range of physical and mathematical methodologies fed into each other. This is best appreciated by a looking at the different 'steps along the way', starting with the pioneering work by Brueckner and collaborators, which was just as necessary and important as the insightful, masterly improvements to Brueckner's approach by Hans Bethe and his students. The main achievement in the work of Brueckner and Bethe et al. was the 'taming' of the hard core of the nucleon-nucleon potential, which has since been understood to result from the exchange of the $\omega$-meson, a 'heavy' photon. The off-shell effects which bedevilled Bethe's work that ended up in the 1963 Reference Spectrum Method were treated relatively accurately by introducing an energy gap between initial bound states and intermediate state. Kuo and Brown 
showed that this would be accurately handled by taking the intermediate states to be free; i.e. by just using Fourier components, as now done in the effective field theory resulting from the renormalization group formalism.

Well, one can say to the young people that this is 'much ado about nothing'. In fact, long ago, when Gerald E. Brown was Professor at Princeton, Murph Goldberger (turning on its head Winston Churchill's famous quote about the R.A.F. during the Battle of Britain) claimed in reference to the nuclear interaction that 'never have so many contributed so little to so few.' Admittedly, at the time it was hard going.

If we had a unique set of interactions, one for each angular momentum, spin and isospin channel, it could be argued that it would be justified to stop there. However, since Brueckner came on the scene, Bethe reorganized the theory, Kuo and Brown wrote their paper that prepared the effective field theory by using the Scott-Mozskowski separation method, and chiral invariance hit the scene. Chiral invariance does not do anything for Yukawa's pion exchange, because the pion gets most of its mass from somewhere outside of the low-energy system, maybe by coupling to the Higgs boson. But the masses of the other mesons drop with increasing density, like

$m_{\rho}^{*} \cong m_{\rho}\left(1-0.2 n / n_{0}\right) \quad$ "Brown/Rho scaling"

where $n$ is the density and $n_{0}$ is nuclear matter saturation density. The change in masses of the scalar- $\sigma$ and vector- $\omega$ mesons pretty much cancel each other in effectsthe scalar exchange giving attraction and the vector repulsion. However, in the tensor force, the $\rho$-exchange 'beats' against the pion exchange, the former cancelling more and more of the latter as the density increases. This decrease with density of the tensor force interaction has important effects:

1. It is responsible for saturation in the nuclear manybody system.

2. It converts an around hour-long carbon-14 lifetime from a superallowed transition in the Wigner $S U(4)$ for $p$-shell nuclei into an archeologically long 5,700year transition.

'Brown/Rho scaling' is also important for neutron stars and may play an important role in turning them into black holes and for 'cosmological natural selection'. It must be admitted that the same effects could be given by three-body forces, but Brown/Rho scaling has a deep connection with chiral symmetry restoration. We shall review these facets in detail.

Undoubtedly, much more is to come, but we believe that now is a good time to summarize the interesting history of the nucleon-nucleon interaction."

Indeed Gerry has devoted a large portion of his research life to nuclear physics, especially to questions related to the nucleon-nucleon interaction and nuclear many-body problem. He has also devoted a large portion of his life to guiding, helping and taking care of his students, postdocs and colleagues (including both of us). Gerry had two operations in 2008, and was not in good health afterwards. With Gerry ill, we worked hard together with Sabine to finish the book, which was published by WS in early 2010. Many of Gerry's friends and colleagues visited him regularly while he was recuperating. I (TTSK) and my wife Annette also visited him often (about once or more each month), and it happened that we saw him in the afternoon of May 27, 2013, just four days before he passed away. He was particularly cheerful that afternoon, smiling, tasting a pastry and making a typical Gerry-style joke. In the following, let us describe briefly the two research projects we have worked on together.

\section{CORE POLARIZATION}

In September 1964, I (TTSK) went to Princeton as an instructor (which is a research associate with minor teaching duties) to work with Gerry. I only learned much later that Gerry knew my advisors Elizabeth Baranger (Univ. of Pittsburgh) and Michel Baranger (CarniegeMellon Univ.) very well, and that they had arranged for me to work with Gerry. In fact, they were all close associates of Hans Bethe. I went to the Palmer Physical Laboratory one day and met Gerry for the first time. I still remember well when he introduced Chun-Wa Wong to me and said "let me introduce my secret weapon to you" (so I realized from that first interaction that Gerry had a good sense of humor and liked to make jokes). Chun-Wa was also a research associate of Gerry; he was a graduate student at Harvard and completed his thesis with Gerry in Copenhagen.

I (JWH) had a similar experience. I met Gerry for the first time in the spring of 2003 when I was a firstyear graduate student at Stony Brook University. My brother Jason (who was already working in the nuclear theory group with TTSK) introduced me one day to Gerry, whose first words to me came as a surprise: "Is your middle name William?" After I answered "yes", Gerry smiled and said "My grandson's name is Jeremy William. Why don't you come work with me this summer?" That was the easiest job interview of my life, but the challenging part came later as I tried to keep up with Gerry's diverse research interests, from hadronic physics, to nuclear astrophysics, to low-energy nuclear structure theory. Soon afterwards, I began to focus on BR scaling [19] and CP [12].

At Princeton Gerry had a rather large and active nuclear theory group. Senior faculty members were Gerry and Ben Bayman. To the best of my (TTSK) memories, the research associates were (in alphabetical order, here and later) J. Blomquist, J. Flores, W. Friedman, 
A.M. Green, A. Kallio, T.T.S. Kuo, H. Picker, A. Lande, P. Mello, G. Ripka, C.W. Wong, and L. Zamick. Visiting faculty members were A. Arima, L. Castillejo, I. Talmi, H. McManus, M. Moshinsky, H. Lipkin and P. Zilser. Gerry had about ten graduate students during his four-year stay at Princeton. I only remember a few of them, namely G. Bertsch, M.Y. Chen, W. Gerace, H. Mavromatis, J. Noble and I. Sharon. I remember Gerry once said "Bertsch was too fast: I gave him a problem and he would disappear for a couple of weeks and come back with the solution. So I soon ran out of problems. I let him graduate." Princeton also had a very active nuclear experimental group, which consisted of (as far as I remember) R. Sherr, J. McCullen, O. Ames and G. Garvey.

Gerry's nuclear theory group worked closely with the Rutgers nuclear physics group, which was a large group with G. Temmer, A. Covello, G. Sartoris and others. Every Monday afternoon we went to Rutgers (about a 20mile drive from Princeton) to attend their weekly seminar. Every Thursday evening they came to Princeton's 'bull session', a Gerry specialty. Usually we all went to have dinner together, and then came to the seminar room at about $7 \mathrm{pm}$, starting the bull session which was an informal seminar with lots of discussions and 'no-timelimit'. Typically it ran for about 3 hours or more till about $11 \mathrm{pm}$. In those years, computers were still 'primitive'; we used card punchers to punch cards and submit jobs (boxes of computing cards) at the computing center. (Gerry often mentioned that in his graduate-student days the computers used paper tapes as inputs.) So after the bull session, the 'young' postdocs almost all first drove to the computing center and submitted some jobs before going home.

The so-called Kuo-Brown matrix elements [2-4] were first developed at that time in order to provide a microscopi basis for the nuclear shell model. We shall only briefly describe them, as more detailed discussions about them have been given by Osnes and by Coraggio (see contributions by them in this memorial volume). The $\mathrm{NN}$ interaction and the nuclear many-body problem are both difficult problems. Gerry recently wrote in his book [1]:

"One of the authors, Gerry Brown, arrived at Princeton in early September, 1964. The next morning, as he came to the Palmer Physics Laboratory, Eugene Wigner, who just preceded him, opened the door for him (It was a real contest to get ahead of Eugene and open the door for him which very few succeeded in doing.) Eugene asked Gerry, as he went into the building, what he planned to work on. 'I plan to work out the nucleon-nucleon interaction in nuclei.' Eugene said that it would take someone cleverer than him, to which Gerry replied that they probably disagreed what it meant to 'work out'. Gerry wanted to achieve a working knowledge, sufficiently good to be able to work out problems in nuclear physics..."

It indeed turned out to be very hard to 'work out' the nucleon-nucleon (NN) interaction in nuclei in a fundamental way, and a more feasible and physically-motivated approach is to compute instead an 'effective' or 'renormalized' nucleon-nucleon interaction. After I (TTSK) arrived Princeton, Gerry asked me to study Brueckner theory [1], which was a new and difficult subject for me at that time. I am still indebted to Chun-Wa for helping me greatly in learning the theory, which was originally designed for nuclear matter but which Gerry intended to apply to finite nuclei in a shell model approach.

Consider as an example the nucleus ${ }^{18} \mathrm{O}$ which has 18 nucleons. In the shell-model effective theory for this nucleus, it is reduced from a many-body problem with 'eighteen' nucleons to a simplified one of just 'two' valence nucleons residing outside of an inert ${ }^{16} \mathrm{O}$ core. This is a simple, smart and bold step, and I think it is of the type of physics that Gerry appreciated. But the two nucleons are in fact renormalized quasi-nucleons, which are different from the original bare ones. The interaction for bare nucleons is $V_{N N}$ while that for the quasi-nucleons is $V_{\text {eff }}$. We first tried $V_{\text {eff }}=G$ where $G$ is the Brueckner G-matrix and used it to calculate the low-lying spectra of ${ }^{18} \mathrm{O}$ and ${ }^{18} \mathrm{~F}$. The calculated spectra were however in poor agreement with experiment, so Gerry then suggested that we take the effective interaction as

$$
V_{e f f}=G+G_{3 p 1 h},
$$

where $G$ represents the direct interaction of the valence nucleons via a $G$-matrix interaction. The term $G_{3 p 1 h}$ denotes the second-order core-polarization diagram shown in Fig. 1(a).

It was to our great joy that the inclusion of $G_{3 p 1 h}$ greatly improved our results. For example, it significantly lowered the lowest $0^{+}$as well as raised a group of high-lying states of ${ }^{18} \mathrm{O}$, making the calculated spectrum in good agreement with experiment. The matrix elements based on $G+G_{3 p 1 h}$ are generally referred to as the Kuo-Brown (KB) matrix elements [1-4] and have been widely used in nuclear shell model calculations for decades with remarkably successful results (see for example Refs. [5-7]).

NN interactions are short-ranged as is the $G$-matrix interaction. In the 1960s it was found mainly in Copenhagen (led by Bohr and Mottelson) that to describe empirical nuclear properties we need also a long-range effective $P_{2}$-force of the form [1, [8]

$$
V_{P_{2}}=-\chi \Sigma_{i j} r_{i}^{2} r_{j}^{2} Y_{2, m}\left(\theta_{i}, \phi_{i}\right) Y_{2,-m}\left(\theta_{j}, \phi_{j}\right)(-1)^{m} .
$$

This empirical force was in fact well reproduced by the core-polarization diagram $G_{3 p 1 h}[\underline{3}$ ], which allowed two nucleons far from each other to interact indirectly through excitations of the core. I (TTSK) remember well a cartoon-like picture of the core polarization effect drawn by Gerry and Akito Arima, where two satellites orbit near the earth surface. They are far away from each other on opposite sides of the earth, so that they 


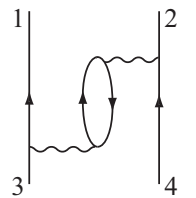

(a)

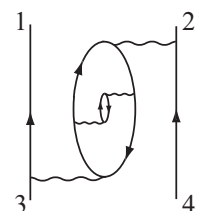

(b)

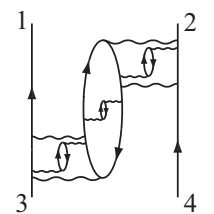

(c)
FIG. 1: Core polarization diagrams.

can hardly interact with each other directly. But they can interact with each other via the tidal waves induced by them.

The success of the Kuo-Brown interactions has led to a number of further studies 9 11], but since the KB core polarization diagram is only a second-order one, a natural question remained: how significant are the higher-order diagrams?

This is a very important question, and we (the Holt brothers, former student Scott Bogner, TTSK and Gerry) have indeed made extensive efforts in answering it as reported in Ref. [12]. In comparison with our earlier calculation [1], we have made several improvements: (i) We employ the renormalization group (RG) low-momentum nucleon-nucleon interaction $V_{l o w-k}$ [13 19], (ii) folded diagrams [20, 21] are summed to all orders, and (iii) an induced-interaction approach is used where particleparticle and particle-hole vertex functions are calculated self-consistently [12].

Microscopic nuclear many-body calculations using realistic $V_{N N}$ interactions are complicated by the difficulties caused by strong repulsive cores normally found in such interactions. For many years, a standard procedure to overcome such difficulties has been the Brueckner $G$ matrix method, where $V_{N N}$ is converted to a smooth $G$-matrix effective interaction by summing ladder diagrams to all orders in the nuclear medium. However, in many ways $G$ is not convenient for many-body calculations. First, its Pauli exclusion operator is complicated for calculation, and second $G$ is energy dependent in an off-energy-shell manner. These features complicate the calculation of diagrams with the $G$-matrix interaction, especially for high-order diagrams such as diagrams (b) and (c) of Fig. 1.

The low-momentum NN interaction $V_{l o w-k}$ is based on a renormalization group approach where one integrates out momentum components beyond a decimation scale $\Lambda$ [13 19]. Briefly speaking, it is given by a pair of $T$-matrix equivalence relations:

$T\left(k^{\prime}, k, k^{2}\right)=V_{N N}\left(k^{\prime}, k\right)+P \int_{0}^{\infty} q^{2} d q \frac{V_{N N}\left(k^{\prime}, q\right) T\left(q, k, k^{2}\right)}{k^{2}-q^{2}}$,

$$
\begin{aligned}
& T\left(p^{\prime}, p, p^{2}\right)=V_{l o w-k}\left(p^{\prime}, p\right) \\
& +P \int_{0}^{\Lambda} q^{2} d q \frac{V_{l o w-k}\left(p^{\prime}, q\right) T\left(q, p, p^{2}\right)}{p^{2}-q^{2}},\left(p^{\prime}, p\right) \leq \Lambda(4)
\end{aligned}
$$

where $P$ denotes the principal value integral. From the above equations, $V_{l o w-k}$ can be obtained from $V_{N N}$. Note that $V_{l o w-k}$ is energy independent and thus convenient for many-body calculations. There are a number of high precision models for $V_{N N}$ [22 28], but their $\left\langle k\left|V_{N N}\right| k^{\prime}\right\rangle$ matrix elements are in fact significantly different from each other although they all reproduce the experimental two-nucleon data quite well [18]. An amazing feature of the different $V_{l o w-k}$ derived from the above different $V_{N N}$ potentials is that they are nearly identical to each other for $\Lambda \lesssim 2.0 \mathrm{fm}^{-1}$, leading to a nearly universal low-momentum NN interaction [18]. Realistic NN potentials are all constructed to fit the experimental NN phase shifts up to $E_{l a b} \leq 350 \mathrm{MeV}$ which corresponds to $\Lambda \simeq 2 \mathrm{fm}^{-1}$, providing an explanation for why $V_{l o w-k}$ with this decimation scale should be nearly universal.

In our new CP calculation [12], we employed a foldeddiagram expansion [20, 21] which provides a formally exact method for calculating the effective interaction $V_{\text {eff }}$ for valence nucleons outside a closed core. It is of the form

$$
V_{e f f}=\hat{Q}-\hat{Q}^{\prime} \int \hat{Q}+\hat{Q}^{\prime} \int \hat{Q} \int \hat{Q}-\hat{Q}^{\prime} \int \hat{Q} \int \hat{Q} \int \hat{Q}+\ldots
$$

where each $\int$ symbol represents a 'fold'. Each $\hat{Q}$-box represents a collection of irreducible diagrams as shown by the diagrams of Fig. 1 . The $\hat{Q}^{\prime}$-box is the same as $\hat{Q}$-box except it starts from the second-order diagrams, namely $\hat{Q}^{\prime}=\hat{Q}-V_{N N}$.

As is well known, high-order CP calculations are difficult to perform, largely because the number of diagrams grows rapidly as one goes to higher orders in perturbation theory. The number of diagrams at third order is already quite large, though still manageable [9 11, 30], but it was soon realized that an order-by-order calculation of CP diagrams beyond third order is not practicable.

To fully assess the effects of core polarization to high order, a non-perturbative method is called for. The nonperturbative method we use is based on the elegant and rigorous induced interaction approach of Kirson 29] and Babu and Brown [31], hereafter referred to as KBB. Other successful non-perturbative summation methods have been developed, such as the parquet summation 32 . and the coupled cluster expansion [33]. In the KBB formalism the vertex functions are obtained by solving a set of self-consistent equations, thereby generating CP diagrams to all orders such as diagrams (b) and (c) of Fig. 1.

Let us now give a brief summary of our all-order calculation for the shell-model effective interaction $V_{\text {eff }}$. We first use $V_{l o w-k}$ to calculate the $\hat{Q}$-box. And in this $\hat{Q}$-box the bubble-in-bubble CP diagrams, like those shown in Fig. 1, are included to all orders in a self-consistent way. Then we obtain $V_{\text {eff }}$ by summing up the $\hat{Q}$-box foldeddiagram series of Eq. (5). To illustrate our results, we show in Fig. 2 a comparison of the $s d$-shell second-order $\mathrm{CP}$ matrix elements with those given by the all-order KBB calculation. The two groups of matrix elements are 


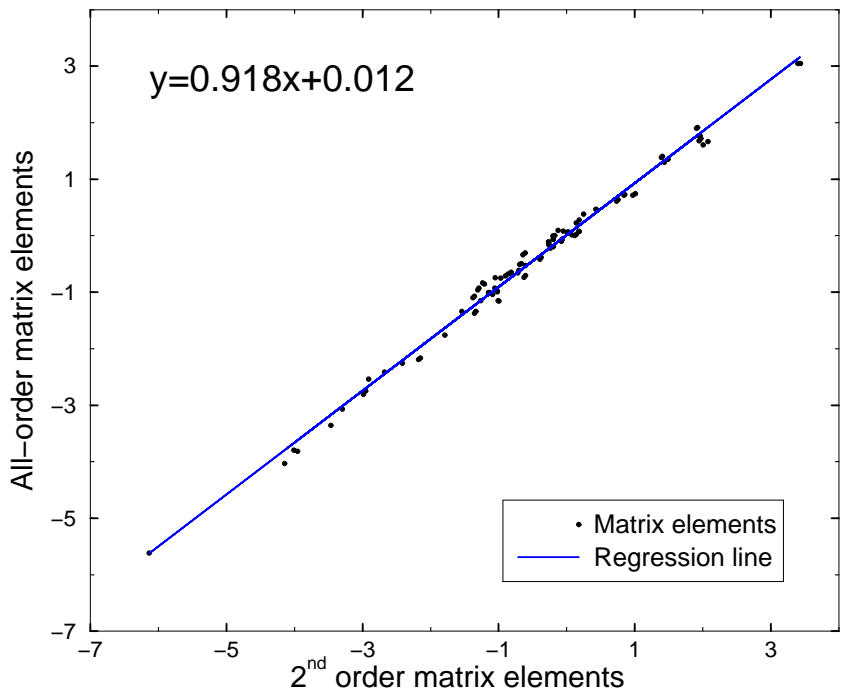

FIG. 2: A comparison of the second-order core polarization matrix elements with those of the all-order KBB calculation.

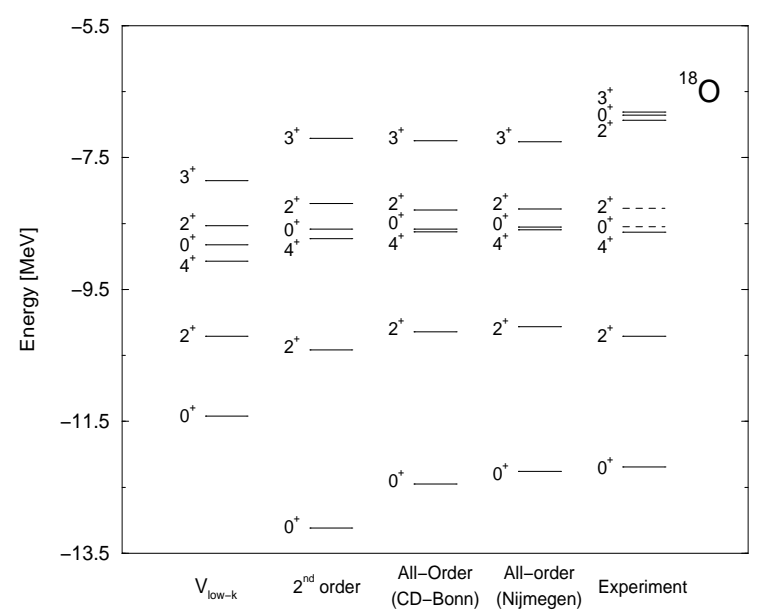

FIG. 3: Spectra for the ${ }^{18} \mathrm{O}$ system calculated to different orders in perturbation theory. Dashed lines for the experimental levels [34] indicate levels with large intruder state mixing 6., 7].

rather close to each other with the all-order elements being about $10 \%$ weaker. A similar comparison for the ${ }^{18} \mathrm{O}$ spectra is given in Fig. 3. The spectrum given in the ' $V_{\text {low }-k}$ ' column is obtained with the $\hat{Q}$-box composed of the first-order diagram only, and consequently the resulting spectrum is too compressed compared to experiment. The spectrum given by the ' 2 nd order' column is obtained with the $\hat{Q}$-box composed of the first- and second-order diagrams. The inclusion of the KBB CP diagrams in the $\hat{Q}$-box largely improves the agreement of the resulting spectra (labeled 'all-order') with experiment.

\section{BROWN-RHO SCALING}

Gerry moved to Stony Brook in 1968 and set up a large and very active nuclear theory group. Faculty members in his group were initially Akito Arima, Andy Jackson and TTSK. There were indeed a large number of visitors and postdocs during Gerry's first years at Stony Brook. Gerry took very good care of them, often putting them up in his home (so that, as Gerry would say, 'they will work day and night'). As far as TTSK can remember, the visitors and postdocs included S. Backman, D. Bes, J. Blomqvist, R. Broglia, M. Chemtob, K. Dietrich, J. Durso, P. Ellis, A. Fessler, B. Friman, H. Gayer, E. Hajimichael, G. Hering, M. Ichimura, L. Ingber, M. Kawai, D. Kurath, R. Lawson, H.K. Lee, G.L. Li, Z.X. Li, Z.Y. Ma, R. Machleidt, H. Muether, E. Nyman, F. Osterfeld, E. Oset, E. Osnes, H. Pauli, Dan-Olaf Riska, M. Rho, J.P. Shen, R. Silbar, H.Q. Song, J. Speth, D. Strottman, K. Suzuki, J. Vergadoes, N. VinhMau, R. VinhMau, J. Wambach, W. Weise, H.F. Wu, S.S. Wu, S.D, Yang, Z.Y. Zhang ...

Having a large number of physicists working together was very pleasant and productive, leading to many longterm collaborations. Mannque Rho was a frequent visitor, and it was at Stony Brook where Brown-Rho scaling [35, 37] originated.

Realistic nuclear potentials are mediated by the exchange of mesons such as the $\pi$-, $\rho$-, $\omega$ - and $\sigma$-meson. In constructing these potentials the meson-nucleon coupling constants are adjusted to fit the 'free-space' NN scattering data. Mesons in a nuclear medium, however, can have properties (masses and couplings) that are different than in free space, as the former are 'dressed' or 'renormalized' by their interactions with the medium. Thus, the NN potential in medium, denoted by $V_{N N}($ med $)$, should be different from that in free-space.

How to obtain $V_{N N}(m e d)$ is of course a most difficult and challenging problem, at least to most of us. Gerry was well known for his physics intuition as well as his brilliant ideas in making complicated problems simple. His Brown-Rho scaling is a typical example. A main result of the well-known Brown-Rho (BR) scaling is $35-$ 37.

$$
\begin{aligned}
& \frac{m_{\sigma}^{*}}{m_{\sigma}} \simeq \frac{m_{N}^{*}}{m_{N}} \simeq \frac{m_{\rho}^{*}}{m_{\rho}} \simeq \frac{m_{\omega}^{*}}{m_{\omega}} \simeq \Phi_{B R}(n), \\
& \Phi_{B R}(n)=1-C \frac{n}{n_{0}}
\end{aligned}
$$

where $m^{*}$ and $m$ denote respectively the in-medium and in-vacuum mass. Here the parameter $C$ has the value $0.15-0.20, n$ is the density of the nuclear medium, and $n_{0}$ is nuclear matter saturation density $\left(0.16 \mathrm{fm}^{-3}\right)$. It is remarkable that the above simple scaling law, derived in the context of chiral symmetry restoration in dense matter, would have dramatic consequences for traditional nuclear structure physics. We shall denote the above 
linear scaling as the BR scaling. This scaling naturally renders $V_{N N}$ a density dependent interaction $V_{N N}(n)$.

Before discussing the various effects of BR scaling, let me (TTSK) first recall a conversation with Gerry many years ago, probably in 1964 when I attended Gerry's Nuclear Physics course at Princeton. He talked a lot about Brueckner theory and also about the empirical Skyrme effective interaction [8] of the form

$$
\begin{aligned}
V_{\text {skyrme }} & =V_{s k}\left(\vec{r}_{1}-\vec{r}_{2}\right)+D_{s k}\left(\vec{r}_{1}-\vec{r}_{2}\right), \\
D_{s k} & =\frac{1}{6}\left(1+x_{3} P_{\sigma}\right) t_{3} \delta\left(\vec{r}_{1}-\vec{r}_{2}\right) n\left(\vec{r}_{a v}\right),
\end{aligned}
$$

where $V_{s k}$ is a two-body $\delta$-function force and $D_{s k}$ is a density-dependent two-body interaction. It was a bit 'strange' that there was a piece of interaction which was density dependent. One day Gerry mentioned that it would be nice to work out a connection between the empirical Skyrme interaction and meson-exchange NN interactions. At that time, my understanding of them was minimal I have to confess, and I was really unable to pursue the matter further. But now there are, I think, indications [46, 47] that BR-scaling may provide a microscopic foundation for the density-dependent Skyrme effective interaction.

We have carried out several studies on the effects of BR scaling on finite nuclei, nuclear matter and neutron stars [42, 43, 45 48]. Let us just briefly describe a few of them. For convenience in implementing BR scaling, we have employed the BonnA and/or BonnS one-bosonexchange potentials [23, 25] whose parameters for $\rho, \omega$ and $\sigma$ mesons are scaled with the density (in our calculations the meson masses and cut-off parameters are equally scaled). Note that $\pi$ is protected by chiral symmetry and is not scaled. That we scale $\rho$ but not $\pi$ has an important consequence for the tensor force, which plays an important role in the famous Gamow-Teller (GT) matrix element for the ${ }^{14} C \rightarrow{ }^{14} N \beta$-decay [43]. The tensor force from $\pi$ - and $\rho$-meson exchange are of opposite signs. A lowering of only $m_{\rho}$, but not $m_{\pi}$, can significantly suppress the net tensor force strength and thus largely diminish the GT matrix element. In addition, the scaling of the $\omega$ meson introduces additional short-distance repulsion into the nucleon-nucleon interaction, which was found to also contribute to the suppression [44]. With BR-scaling we were able to satisfactorily reproduce the $\sim 5800$-yr long lifetime of this decay; I remember well that Gerry was very pleased with this result.

BR scaling has been applied to several nuclear matter calculations [42, 45 48]. Let me start from a result, as shown in Fig. 4 of Ref. [48], to illustrate the current situation. This calculation employed a so-called newBrown-Rho (new-BR) scaling [4] which is based on a half-Skyrmion model to be described briefly later. The equation of state (EOS) labeled (C) is obtained from $V_{\text {low }-k}$ (derived from the BonnS $V_{N N}$ [25]) without any BR scaling. It does not exhibit satisfactory saturation properties. It is a general result that $V_{N N}$ alone cannot give satisfactory nuclear saturation properties as illustrated by $(\mathrm{C})$ of Fig. 4.

We have found that Brown-Rho scaling improves this situation dramatically [42, 45-48]. Moreover the combined potential given by the sum of the unscaled- $V_{N N}$ and $D_{s k}$, the latter being the Skyrme density-dependent force of Eq. (7), can also give equally satisfactory nuclear matter saturation properties [46]. Four such EOS's are shown in Fig. 5, where $\Lambda$ denotes the decimation momentum scale for $V_{\text {low }-k}$. As seen, the four EOS's agree with each other closely, all giving $E_{0} / A \simeq-15 \mathrm{MeV}$, $k_{F} \simeq 1.40 \mathrm{fm}^{-1}$ and $K \simeq 150 \mathrm{MeV}$. Thus to have satisfactory nuclear matter saturation properties, we may use either a BR-scaled $V_{N N}$ or an unscaled- $V_{N N}+D_{s k}$; this indicates that a microscopic foundation for the empirical Skyrme density-dependent force may be provided by BR scaling.

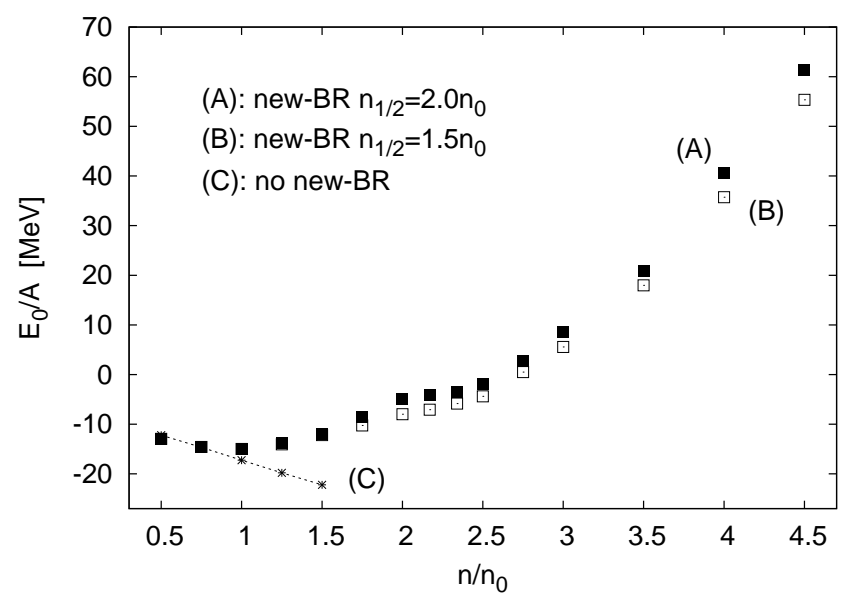

FIG. 4: Comparison of the EOS for symmetric nuclear matter calculated with and without the new-BR scaling. Transition densities of $n_{1 / 2}=2.0$ (solid square) and $1.5 n_{0}$ (open square) are employed. See text for more explanations.

We now describe the new-BR scaling [48] on which the results shown in Fig. 4 are based. The idea behind this scaling is that when a large number of skyrmions as baryons are put on an FCC (face-centered-cubic) crystal to simulate dense matter, the skyrmion matter undergoes a transition to a matter consisting of half-skyrmions [38] in $\mathrm{CC}$ configuration at a density that we shall denote as $n_{1 / 2}$. This density is difficult to pin down precisely but it is more or less independent of the mass of the dilaton scalar, the only low-energy degree of freedom that is not well-known in free space. The density at which this occurs has been estimated to lie typically between 1.3 and 2 times normal nuclear matter density $n_{0}$ [39]. In our model, nuclear matter is separated into two regions I and II respectively for densities $n \leq n_{1 / 2}$ and $n>n_{1 / 2}$. As inferred by our model, they have different scaling functions

$$
\Phi_{i}(n)=\frac{1}{1+C_{i} \frac{n}{n_{0}}}, \quad i=I, I I
$$




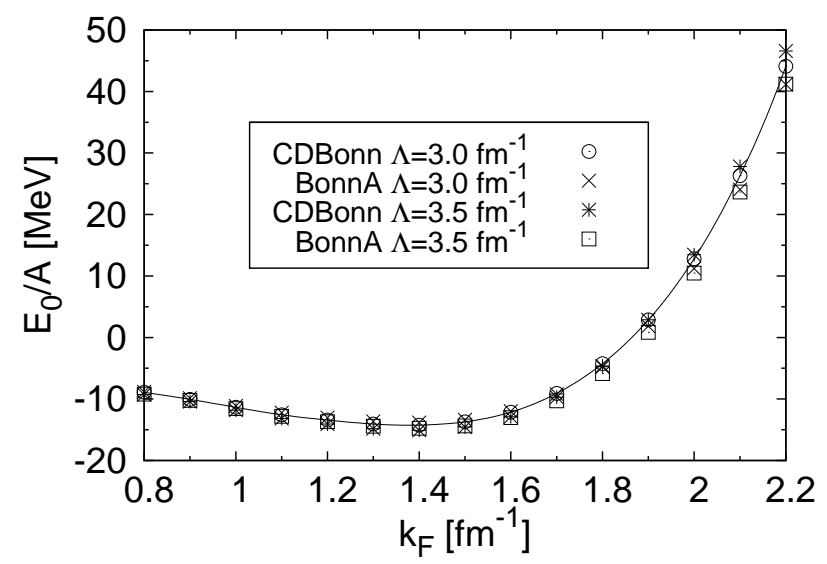

FIG. 5: Ring-diagram EOS for symmetric nuclear matter with the interaction being the sum of $V_{l o w-k}$ and the Skyrme density dependent force of Eq.(7). Four sets of results are shown for CDBonn and BonnA potentials with $\Lambda=3$ and $3.5 \mathrm{fm}^{-1}$. A common Skyrme force of $t_{3}=2000 \mathrm{MeV}-f m^{6}$ and $x_{3}=0$ is employed.

The above two-region scaling is the new-BR scaling mentioned earlier.

The EOS (A) and (B) of Fig. (4) are obtained with the new-BR scaling with $n_{1 / 2}=1.5$ and $2.0 n_{0}$ respectively. As described in [48], we employ in our new-BR calculations the BonnS potential [25] with scaling parameters $C_{\rho}=0.13, C_{\sigma}=0.121, C_{\omega}=0.139, C_{N}=0.13$ and $C_{g, \rho}=C_{g, \omega}=0$ for region I. For region II the scaling parameters are $C_{\rho}=0.13, C_{\sigma}=0.121, C_{\omega}=0.139, C_{g, \rho}=0.13$, $C_{g, \omega}=0$ and $m_{N}^{*} / m_{N}=y(n)=0.77$. Note that this scaling has some special features: In region I the coupling constants $g_{\rho N}$ and $g_{\omega N}$ are not scaled, while in region II only the coupling constant $g_{\rho N}$ is scaled. Also in region II the nucleon mass is a density-independent constant $\left(m_{N}^{*} / m_{N}=0.77\right)$. Note that our choices for the $C$ parameters are consistent with the Ericson scaling which is based on a scaling relation for the quark condensate $\frac{\langle\bar{q} q\rangle^{*}}{<\bar{q} q>}[40]$. According to this scaling, at low densities one should have $C \simeq D / 3$ with $D=0.35 \pm 0.06$.

We note that the above scaling is only 'inferred' by our Skyrmion-half-Skyrmion model [48]. As a first step to check this scaling, we have carried out several applications. In Fig. 4, the calculated EOS for symmetric nuclear matter using transition densities $n_{1 / 2}=1.5(\mathrm{~A})$ and $2.0 n_{0}$ (B) are shown. Both give an energy per nucleon $E_{0} / A=-15 \mathrm{MeV}$, saturation density $k_{F}=1.30 \mathrm{fm}^{-1}$ and compression modulus $K=208 \mathrm{MeV}$, all in satisfactory agreement with the empirical values [48]. We believe that our scaling works well for low densities of $n \lesssim 1.5 n_{0}$.

How to scale the mesons at densities beyond $n_{0}$ is still an open question. By way of heavy-ion collision experiments, there has been much progress in determining the nuclear symmetry energy $E_{\text {sym }}$ up to densities as high as $\sim 5 n_{0}$ [49 51]. Thus an application of our new-BR scaling to the calculation of $E_{\text {sym }}$ would provide an im- portant test for this scaling in the region with $n>n_{1 / 2}$. As displayed in Fig. 6, our calculated symmetry energies agree well with the experimental constraints [49, 51].

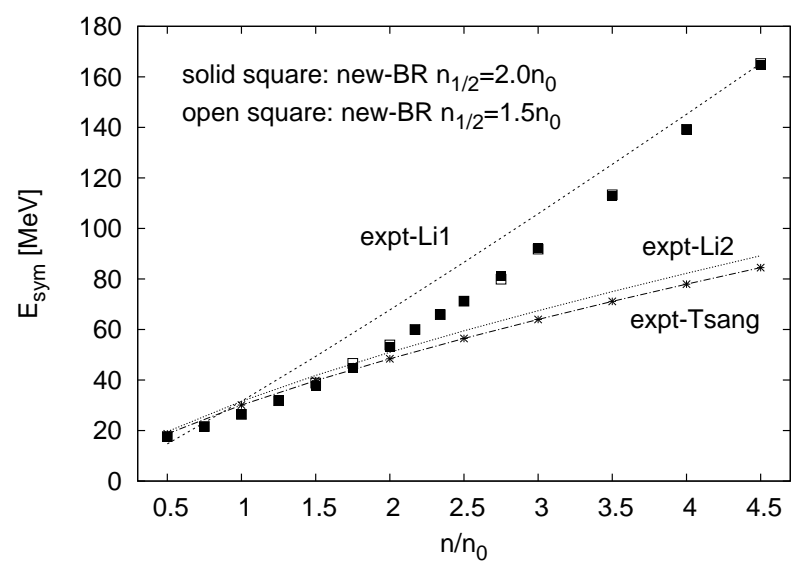

FIG. 6: Comparison of our calculated nuclear symmetry energies with the empirical upper (expt-Li1) and lower (exptLi2) constraints of $\mathrm{Li}$ et al. 49] and the empirical results of Tsang et al. (expt-Tsang) [51].

The EOS at high densities $\left(n \simeq 5-10 n_{0}\right)$ is important for neutron-star properties. Thus an application of the new-BR scaling to neutron star structure would provide a useful test. As shown in Fig. 7, our calculated neutronstar maximum mass is about $2.4 M_{\odot}$, slightly larger than the empirical value of $\sim 2 M_{\odot}$ [48]. In our calculations, the central densities of neutron stars are $\sim 5 n_{0}$. At such densities, how to scale the hadrons with the medium remains to be an interesting and open question. Much remains to be done.

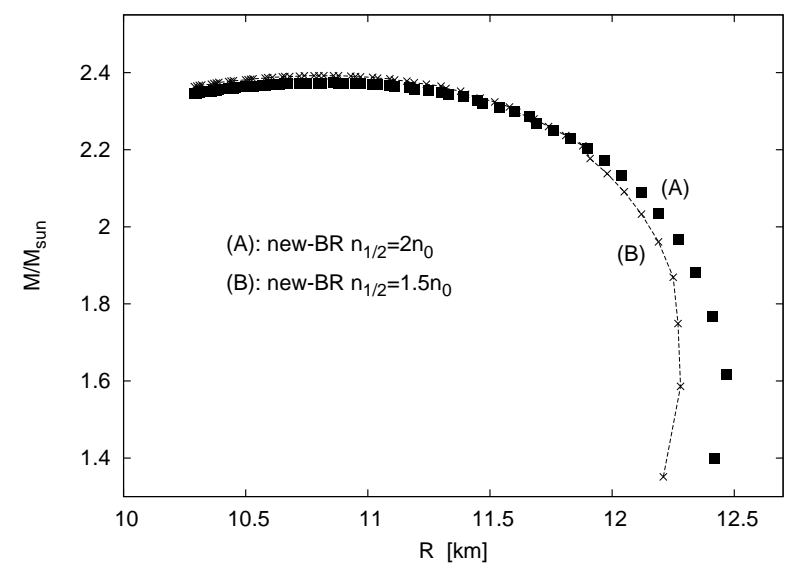

FIG. 7: Mass-radius trajectories of neutron stars calculated with new-BR scalings using $n_{1 / 2}=2.0(\mathrm{~A})$ and $1.5 n_{0}(\mathrm{~B})$. The maximum neutron-star mass and its radius for these two cases are respectively $\left\{M_{\max }=2.39 M_{\odot}\right.$ and $\left.R=10.90 \mathrm{~km}\right\}$ and $\left\{M_{\max }=2.38 M_{\odot}\right.$ and $\left.R=10.89 \mathrm{~km}\right\}$. 


\section{SUMMARY}

It is indeed our fortune and privilege to have met Gerry and worked with him since our early careers. He was not only a great scientist but also a kind person, who supported his colleagues and students over many years. I (TTSK) especially have many fond memories of fun times together, starting in 1964 when I met Gerry at Princeton, and our relationship extended beyond academics (such as our regular tennis matches). Gerry's insights into core polarization and Brown-Rho scaling are of fundamental importance for our understanding of effective nucleonnucleon interactions in a nuclear medium and the possible connections to chiral symmetry restoration in dense matter. It was a pleasure to explore these topics together, and we have learned much from him over the years. Let us express our deep gratitude to him.

Acknowledgement We are grateful to M. Rho and R. Machleidt for helpful discussions. This work was supported in part by the Department of Energy under Grant No. DE-FG02-88ER40388 and DE-FG02-97ER-41014.

[1] G.E. Brown, T.T.S. Kuo, J.W. Holt and Sabine Lee (editors), 'The Nucleon-Nucleon Interaction and the Nuclear Many-Body Problems-selected papers of Gerald Brown and T.T.S. Kuo', World Scientific Pub. Co. (2010).

[2] T. T. S. Kuo and G. E. Brown, Nucl. Phys. 85 (1966) 40.

[3] G. E. Brown and T. T. S. Kuo, Nucl. Phys. A92 (1967) 481.

[4] T. T. S. Kuo and G. E. Brown, Nucl. Phys. A114 (1968) 241-279.

[5] A. Poves and A. P. Zucker, Phys. Rep. 70 (1981) 562.

[6] B.H. Wildenthal, Prog. Part. Nucl. Phys. 11 (1984) 5.

[7] B.A. Brown and B.H. Wildenthal, Ann. Rev. Nucl. Part. Sci. 38 (1988) 29.

[8] 'The Nuclear Many-Body Problem', P. Ring and P. Schuck, Springer-Verlag (1980).

[9] B. R. Barrett and M. Kirson, Nucl. Phys. A148 (1970) 145.

[10] M. Jorth-Jensen, T.T.S, Kuo and Eivind Osnes, Physics Reports 261 (1995) 205.

[11] L. Coraggio, A. Covello, A. Gargano, N. Itako and T.T.S. Kuo, Prog. Part. Nucl. Phys. 62 (2009) 135.

[12] J. D. Holt, J. W. Holt, T. T. S. Kuo, G. E. Brown and S. K. Bogner, Phys. Rev. C 72 (2005) 041304(R).

[13] S. K. Bogner, T. T. S. Kuo and L. Coraggio, Nucl. Phys. A684 (2001) 432c.

[14] T. T. S. Kuo, S. K. Bogner, and L. Coraggio, Nucl. Phys. A704 (2002) 107c.

[15] S. K. Bogner, T. T. S. Kuo, L. Coraggio, A. Covello and N. Itaco, Phys. Rev. C 65 (2002) 051301(R).

[16] T. T. S. Kuo, S. K. Bogner, L. Coraggio, A. Covello, and N. Itaco, in Challenges of Nuclear Structure (Proceedings of the 7th International Seminar on Nuclear Structure; Maiori, Italy, May 27-31, 2001), ed. by A. Covello, p. 129, World Scientific Pub. Co. (2002).
[17] A. Schwenk, G. E. Brown, and B. Friman, Nucl. Phys. A703 (2002) 191.

[18] S. K. Bogner, T. T. S. Kuo, and A. Schwenk, Phys. Rep. 386 (2003) 1.

[19] J. D. Holt, T. T. S. Kuo and G. E. Brown, Phys. Rev. C 69 (2004) 034329.

[20] T.T.S. Kuo and E. Osnes, Lecture Notes in Physics Vol.364 (Springer-Verlag 1990) p. 1.

[21] T.T.S. Kuo, S.Y. Lee and K.F. Ratcliff, Nucl. Phys. A176 (1971) 172.

[22] M. Lacombe et al., Phys. Rev. C21 (1980) 861.

[23] R. Machleidt, Adv. Nucl. Phys. 19 (1989) 189.

[24] R. Machleidt, Phys. Rev. C 63 (2001) 024001.

[25] R. Machleidt, 'Computational Nuclear Physics 2-Nuclear Reactions', ed K. Langanke, J. A. Maruhn, S. E. Koonin, (Springer NY, 1993), p. 1.

[26] R. B. Wiringa, V. G. J. Stoks and R. Schiavilla, Phys. Rev. C 51, 38 (1995).

[27] V. G. J. Stoks, R. A. M. Klomp, C. P. F. Terheggen and J. J. de Swart, Phys. Rev. C 49 (1994) 2950.

[28] D. R. Entem andR. Machleidt, Phys. Rev. C 68 (2003) 041001.

[29] M. W. Kirson, Ann. Phys. 66, 624 (1971); ibid. 68, 556 (1971); ibid. 82 (1974) 345.

[30] L. Coraggio, A. Covello, A. Gargano, N. Itaco and T.T.S. Kuo, Ann. Phys. 327 (2012) 2125.

[31] S. Babu and G. E. Brown, Ann. Phys. 78, 1 (1973).

[32] A. Jackson et al., Phys. Rep. 86 (1982) 55.

[33] D. J. Dean et al., Phys. Rev. C 69 (2004) 054320.

[34] D.R. Tilley, H.R. Weller, C. M. Cheves and R.M. Chasteler, Nucl. Phys. A595 (1995) 1.

[35] G. E. Brown and M. Rho, Phys. Rev. Lett. 66 (1991) 2720.

[36] T. Hatsuda and S.H. Lee, Phys. Rev. C 46 (1992) R34.

[37] G. E. Brown and M. Rho, Phys. Rep. 396 (2004) 1.

[38] A.S. Golhaber and N.S. Manton, Phys. Lett. B198 (1987) 231.

[39] B. Y. Park, D. P. Min, M. Rho and V. Vento, Nucl. Phys. A 707 (2002) 381; H. J. Lee et al., Nucl. Phys. A 723 (2003) 427; M. Rho, arXiv:0711.3895 [nucl-th].

[40] M. Ericson, Phys. Lett. B 301 (1993) 11.

[41] J. Gasser, H. Leutwyler and M.E. Sainio, Phys. Lett. B 253 (1991) 252.

[42] J. W. Holt, G. E. Brown, J. D. Holt and T. T. S. Kuo, Nucl. Phys. A785 (2007) 322.

[43] J. W. Holt, G. E. Brown, T. T. S. Kuo, J. D. Holt and R. Machleidt, Phys. Rev. Lett. 100 (2008) 062501.

[44] J. W. Holt, N. Kaiser and W. Weise, Phys. Rev. C 79 (2009) 054331.

[45] L. W. Siu, J. W. Holt, T. T. S. Kuo and G. E. Brown, Phys. Rev. C 79 (2009) 0540004.

[46] H. Dong, T.T.S. Kuo and R. Machleidt, Phys. Rev. C80 (2009) 065803.

[47] H. Dong, T.T.S. Kuo and R. Machleidt, Phys. Rev. C 83 (2011) 054002.

[48] H. Dong, T.T.S. Kuo, H. K. Lee, R. Macleidt and M. Rho, Phys. Rev. C87 (2013) 054332.

[49] B.A. Li and L.W. Chen, Phys. Rev. C72 (2005) 064611.

[50] B. A. Li, L. W. Chen and C. M. Ko, Phys. Rep. 464 (2008) 113.

[51] M.B. Tsang, Yingxun Zhang, P. Danielewicz, M. Faminao, Zhuxia Li, W.G. Lynch and A.W. Steiner, Phys. Rev. Lett. 102 (2009) 122701. 